Ana Carolina Lemos Pereira (iD) https://orcid.org/0000-0002-1198-8961

Heloisa Aparecida Souza ${ }^{a}$ iD https://orcid.org/0000-0002-0297-0858

Sergio Roberto de Luccab (D) https://orcid.org/0000-0001-6023-0949

Aparecida Mari Igutib

(iD) https://orcid.org/0000-0002-1309-7433

a Pontifícia Universidade Católica de Campinas, Faculdade de Psicologia. Campinas, SP, Brasil.

b Universidade Estadual de Campinas, Faculdade de Ciências Médicas. Campinas, SP, Brasil.

Contato:

Ana Carolina Lemos Pereira

E-mail:

lemosacarolina@gmail.com

Os autores informam que a pesquisa foi financiada pelo Conselho Nacional de Desenvolvimento Científico e Tecnológico (CNPq), na modalidade Bolsa de Doutorado - GD, número do processo 141439/2015-7.

Os autores declaram que não há conflito de interesses.

Os autores informam que o trabalho não foi apresentado em evento científico e que foi baseado na tese Saúde mental relacionada ao trabalho: a complexidade das situações de trabalho e as limitações da abordagem dos fatores de riscos psicossociais, de Ana Carolina Lemos Pereira, defendida em 2019 na Faculdade de Ciências Médicas da Universidade Estadual de Campinas.

\section{Fatores de riscos psicossociais no trabalho: limitações para uma abordagem integral da saúde mental relacionada ao trabalho}

\author{
Psychosocial risk factors at work: limitations for a \\ comprehensive approach to work-related mental health
}

\section{Resumo}

Introdução: os riscos psicossociais são apontados como fatores que podem contribuir ou mesmo desencadear estresse, adoecimento físico e mental nos trabalhadores. Objetivo: contribuir com o debate a respeito das limitações dos fatores de riscos psicossociais no trabalho e refletir sobre a necessidade de uma abordagem ampliada e integral na atenção à saúde mental dos trabalhadores. Métodos: estudo qualitativo baseado em levantamento bibliográfico e análise documental. Realiza-se uma breve contextualização do mundo do trabalho, apresentam-se dados sobre o adoecimento mental dos trabalhadores e as diversas concepções dos fatores de riscos psicossociais presentes na literatura internacional, bem como as orientações fornecidas pela legislação brasileira. Discussão: problematizam-se as avaliações psicossociais na forma como têm sido realizadas no Brasil e as limitações do uso de instrumentos quantitativos. Apresenta-se uma abordagem ampliada e integral para a compreensão do adoecimento dos trabalhadores, apontando para a necessidade de se considerar os diversos elementos políticos, econômicos, sociais, culturais, ambientais e intrapsíquicos no processo de desgaste mental da classe trabalhadora.

Palavras-chave: fatores de risco psicossociais; saúde mental; trabalho; saúde do trabalhador.

\begin{abstract}
Introduction: psychosocial risks are identified as factors that can contribute or even trigger stress, physical and mental illness in workers. Objective: to contribute to the debate about the limitations of psychosocial risk factors at work and reflect upon the need for an expanded and comprehensive approach to the workers' mental health care. Methods: a qualitative study based on literature review and document analysis. We carried out a brief contextualization of the world of work, presented data on the workers' mental illness and approached the different conceptions of the psychosocial risk factors found in the international literature, as well as the guidelines provided by the Brazilian legislation. Discussion: we discussed the way psychosocial assessments have been carried out in Brazil and the limitations of making use of quantitative tools. We presented an expanded and comprehensive approach to understand the workers'illness process, pointing at the need to consider the various political, economic, social, cultural, environmental and intrapsychic elements in the mental strain of the working class.
\end{abstract}

Keywords: psychosocial risk factors; mental health; work; occupational health. 


\section{Introdução}

Embora não exista um consenso a respeito do termo "fatores de riscos psicossociais no trabalho (FRPT)", e o conceito seja empregado em diversos contextos laborais ${ }^{1-3}$, de uma maneira geral os riscos psicossociais são apontados como fatores que podem contribuir ou mesmo desencadear estresse, adoecimento físico e mental nos trabalhadores. Alguns autores, como Bernardo et al. ${ }^{4}$, Seligmann-Silva ${ }^{5}$, Laurel e Noriega ${ }^{6}$ e Lacaz $^{7}$, problematizam o uso da concepção de fatores de risco para analisar os elementos psíquicos e sociais existentes no ambiente de trabalho, defendendo uma visão ampla da realidade de trabalho. Nessa direção, o presente ensaio tem o objetivo de contribuir com o debate a respeito das limitações dos fatores de riscos psicossociais no trabalho e refletir sobre a necessidade de uma abordagem ampliada e integral na atenção à saúde mental dos trabalhadores.

Para tal, inicia-se com uma breve contextualização do complexo e dinâmico mundo do trabalho, frequentemente marcado pela precarização, que compromete a saúde, a segurança e a dignidade dos trabalhadores. Em seguida, são apresentados dados sobre o adoecimento mental no trabalho e as diversas concepções dos FRPT presentes na literatura internacional, bem como as orientações fornecidas pela legislação brasileira. Problematizam-se ainda a forma como as avaliações psicossociais têm sido realizadas no Brasil e as limitações de instrumentos quantitativos como única referência de compreensão da realidade laboral.

As críticas ao conceito de FRPT partem da compreensão do binômio saúde/doença como processo ${ }^{6,8}$. Inquestionavelmente, os chamados "fatores de riscos psicossociais" são elementos que podem ser prejudiciais à saúde dos trabalhadores. O que se contesta é a possibilidade de tomar seus determinantes como medidas exatas, aplicáveis a todos os sujeitos de forma generalizada. É preciso considerar as particularidades e a complexidade de cada situação de trabalho. Nesse sentido, indica-se a necessidade de uma visão contextualizada dos elementos envolvidos no processo de adoecimento mental dos trabalhadores.

Vale ainda ressaltar que as reflexões travadas ao longo deste ensaio foram motivadas por experiências empíricas e discussões em grupos de estudos que defendem uma visão integral e contextualizada da relação entre trabalho e adoecimento mental dos trabalhadores.

\section{Breve contextualização do mundo do trabalho}

Ao buscar compreender o adoecimento mental dos trabalhadores, é essencial considerar a precarização que compõe o atual contexto do trabalho no Brasil ${ }^{9-11}$. Fazer uma análise do atual panorama, que inclui a flexibilização, a intensificação do trabalho e a perda de direitos trabalhistas, é tirar uma fotografia de uma realidade em permanente mutação. Se há alguns anos atribuíam-se as mudanças operadas no mundo do trabalho a processos que marcaram a década de 1990, como a reestruturação produtiva e a globalização, agora, perante o cenário político e econômico brasileiro, nos deparamos com uma situação ainda mais complexa, que tem modificado profundamente a forma de (sobre) viver e trabalhar na sociedade capitalista.

Produzir cada vez mais com menos, em tempos mais curtos, é a meta dos atuais modelos de gestão. Sob o discurso de excelência, melhoria contínua e empregados cooptados como "colaboradores", as relações de trabalho são transformadas de forma a mascarar a dominação capitalista sobre o trabalho humano, ao passo que o intensificam cada vez mais $^{12}$. Para Seligmann-Silva ${ }^{5}$, essas novas técnicas de gerenciamento e o discurso da excelência reforçam o individualismo, aumentam o cansaço e se constituem como motor central da produção de danos à saúde mental dos trabalhadores.

Antunes e Praun ${ }^{9}$ afirmam que as corporações transnacionais passaram a impor à classe trabalhadora patamares salariais e condições de existência cada vez mais rebaixadas. A busca por complementação de renda, bem como os altos índices de desemprego, tem levado os trabalhadores a ingressar no modelo que Abílio $^{13}$ denomina "uberização do trabalho"c, caracterizado pela ausência de vínculo empregatício, pela intensificação do trabalho e pela vigilância e controle permanentes. A uberização "refere-se a um novo estágio da exploração do trabalho, que traz mudanças qualitativas ao estatuto do trabalhador, à configuração das empresas, assim como às formas de controle, gerenciamento e expropriação do trabalho"13.

Pode-se afirmar que as crises econômicas, inerentes ao modo de produção capitalista, acentuam e tornam ainda mais evidente a precarização do trabalho, enquanto o aumento do índice de desemprego e a perda de direitos trabalhistas ${ }^{\mathrm{d}}$ fomentam o sentimento

c Termo baseado na proposta de organização de trabalho popularizada pelo aplicativo de transportes privados Uber.

d No ano de 2017, sob o slogan da modernização, foi aprovada a Reforma Trabalhista Brasileira, que promoveu um verdadeiro retrocesso, implicando grandes perdas de direitos para a classe trabalhadora. Em 2019, foi aprovada a Reforma da Previdência, que modificou o tempo de contribuição e idade mínima para aposentadoria, mas omitiu as vultosas dívidas de grupos empresariais para com a Previdência. 
de insegurança, desamparo e adoecimento mental da classe trabalhadora ${ }^{9}$.

Não cabe ao escopo deste ensaio realizar uma análise detalhada do contexto de diminuição de direitos trabalhistas e de aumento da precarização e intensificação do trabalho, entretanto não se pode deixar de considerar esses elementos ao buscar compreender o adoecimento mental dos trabalhadores.

\section{Dados sobre a saúde mental no trabalho}

No Brasil, segundo dados da Previdência Sociale, os transtornos mentais são a terceira causa de afastamentos entre os trabalhadores segurados ${ }^{14}$. Embora apresentem baixa letalidade (pouco mais de 1\% da mortalidade), os transtornos mentais são responsáveis por mais de $12 \%$ da incapacitação decorrente de doenças, percentual que sobe para $23 \%$ em países desenvolvidos. Das dez principais causas de incapacitação, cinco são transtornos psiquiátricos, sendo os principais a depressão (13\%), o alcoolismo $(7,1 \%)$, a esquizofrenia (4\%), o transtorno bipolar $(3,3 \%)$ e o transtorno obsessivo-compulsivo $(2,8 \%)^{15}$.

No Brasil, dados sobre a distribuição da concessão de auxílio-doença relacionado a acidente de trabalho ocasionado por transtornos mentais e comportamentais, conforme as categorias inscritas na Classificação Internacional de Doenças (CID-10), mostram que as "reações ao stress grave e transtorno de adaptação" (F43) têm uma frequência de 31,05\% em 52.974 casos, os episódios depressivos (F32) 27,11\%, e outros transtornos ansiosos (F41) 21,10\% ${ }^{14}$.

O aumento nos índices de afastamento do trabalho devido aos transtornos mentais fez a Organização Internacional do Trabalho (OIT) enfatizar a relevância dos fatores psicossociais e sua influência no desencadeamento do estresse relacionado ao trabalho, posição também defendida posteriormente pela Organização Mundial de Saúde (OMS) ${ }^{16-17}$.

A OIT avalia que os riscos psicossociais e o estresse relacionado ao trabalho conduzem a comportamentos que afetam a saúde, como uso de substâncias psicoativas e consumo abusivo de álcool, podendo ser responsáveis também por distúrbios do sono e excesso de peso ${ }^{18}$.

A instituição alerta ainda sobre uma série de mudanças no mundo do trabalho que tem contribuído para o estresse relacionado ao trabalho, como a globalização e fenômenos a ela associados: demandas por contratos flexíveis, fragmentação do mercado de trabalho, downsizing (redução da empresa com demissão de trabalhadores) e terceirização, contratos temporários e insegurança no trabalho, maior carga de trabalho e aumento da pressão, além de baixo equilíbrio entre a vida pessoal e trabalho ${ }^{19}$. Essas informações da OIT também são apresentadas no boletim produzido pela Previdência Social sobre a concessão de benefícios por incapacidade relacionados a transtornos mentais e comportamentais entre 2012 e $2016^{14}$.

Sabe-se que os casos de adoecimento mental relacionados ao trabalho são com frequência subnotifica$\operatorname{dos}^{5}$, mas as informações acima revelam a gravidade e a urgência de se investir em ações que busquem preservar a saúde mental dos trabalhadores.

\section{Principais bases conceituais dos fatores de riscos psicossociais no trabalho}

A OIT define os fatores psicossociais como uma das principais preocupações do mundo do trabalho contemporâneo. A instituição considera como fatores psicossociais a interação entre ambiente, conteúdo e condições de trabalho, capacidade dos trabalhadores de atender as demandas de trabalho, necessidades e expectativas dos trabalhadores, cultura e fatores pessoais e extralaborais. Dependendo da forma como estes aspectos são percebidos ou vivenciados, eles podem afetar a saúde, o desempenho e a satisfação no trabalho ${ }^{16}$.

O Quadro Europeu de Gestão dos Riscos Psicossociais (PRIMA-EF) ${ }^{17}$, desenvolvido no âmbito da OMS, inclui fatores organizacionais e relacionados ao trabalho como fatores de riscos psicossociais, citando a gestão de recursos humanos, concordância com os objetivos organizacionais, políticas de segurança e saúde ocupacional, formas de comunicação, feedback, autonomia ou latitude de decisão, segurança no emprego, apoio social e horário de trabalho.

Já para o Instituto Sindical de Trabajo, Ambiente y Salud (ISTAS) ${ }^{20}$ da Espanha:

Os riscos psicossociais são condições de trabalho de-
rivadas da organização do trabalho, para as quais se
tem evidência científica suficiente que demonstra que
prejudicam a saúde dos trabalhadores e trabalhado-
ras. PSICO porque nos afetam através da psique (con-
junto de atos e funções da mente) e SOCIAL porque
sua origem é social: determinadas características da
organização do trabalho. (p. 25 , tradução livre)

$\mathrm{O}_{\text {ISTAS }}^{20}$ classifica os riscos psicossociais em quatro grandes grupos: excesso de exigências psicológicas

e Este ensaio traz como referência publicações do Ministério da Previdência Social. No entanto, é importante ressaltar que a pasta foi extinta em maio de 2016, passando a integrar o Ministério do Trabalho e Emprego. Posteriormente, com a extinção do Ministério do Trabalho, Emprego e Previdência Social, em 2019, suas competências foram incorporadas pelo Ministério da Economia. 
do trabalho; falta de influência ou autonomia no desenvolvimento do trabalho; falta de suporte social e baixa ou má qualidade da liderança (que não apoia o trabalhador); e escassas compensações do trabalho.

Segundo Gollac e Bodier ${ }^{21}$, é imprescindível considerar que a origem dos fatores psicossociais de risco está nas relações, condições e organização de trabalho. Esses autores consideram seis dimensões dos fatores psicossociais de risco: intensidade e condições temporais do trabalho, exigências emocionais, autonomia, relações sociais, conflito de valores e insegurança nas situações de trabalho.

Veloso Neto ${ }^{22}$ elenca dez dimensões de fatores de risco psicossocial: as relações laborais; os ritmos e tempos de trabalho; o conteúdo e organização do trabalho; o trabalho emocional; a conciliação entre as esferas da vida; a igualdade no trabalho e emprego; as relações sociais de trabalho; a qualidade da liderança e avaliação de desempenho; a participação dos trabalhadores; e a saúde geral dos trabalhadores.

Já Alcántara e Sánchez ${ }^{23}$ consideram que o conceito de fatores psicossociais tem sido utilizado como referência tanto para elementos externos ao indivíduo quanto para os efeitos que se sucedem nele. Os "elementos externos" são as exigências laborais, as cargas de trabalho, as demandas e os estressores, que geralmente fazem referência às condições de trabalho objetivas a que são submetidos os trabalhadores, e dentre os efeitos destaca-se o estresse enquanto "dano psicossocial". As autoras consideram ainda como sinônimos o conceito de "exigências laborais", "demandas" e "fatores psicossociais".

Álvarez Briceño ${ }^{24}$ define "risco psicossocial" como as características das condições e organização do trabalho que afetam a saúde das pessoas através de mecanismos psicológicos e fisiológicos. $\mathrm{O}$ autor aponta como a legislação venezuelana reconhece os riscos psicossociais e define as responsabilidades administrativas, civil e penal dos empregadores com relação a esses riscos, pontuando ainda a importância de se enfatizar os riscos psicossociais em um contexto preventivo da lei.

No Brasil, Baruki ${ }^{25}$ resgata várias definições dos riscos psicossociais presentes na literatura ao propor um regime jurídico preventivo que considere estes riscos na determinação dos transtornos mentais no trabalho.

A Norma Técnica sobre Lesões por Esforços Repetitivos ou Distúrbios Osteomusculares Relacionados ao Trabalho da Previdência Social ${ }^{26}$ define que:
Os fatores psicossociais do trabalho são as percepções subjetivas que o trabalhador tem dos fatores de organização do trabalho. Como exemplo de fatores psicossociais podemos citar: considerações relativas à carreira, à carga e ritmo de trabalho e ao ambiente social e técnico do trabalho. A "percepção" psicológica que o indivíduo tem das exigências do trabalho é o resultado das características físicas da carga, da personalidade do indivíduo, das experiências anteriores e da situação social do trabalho.

Guimarães ${ }^{27}$ corrobora esta definição e acrescenta que os riscos psicossociais também interferem no comportamento e desempenho dos trabalhadores e podem provocar danos psicológicos.

A Consolidação das Leis do Trabalho (CLT) disciplina e normatiza as condições de trabalho a partir das Normas Regulamentadoras (NRs) do Ministério do Trabalho e Emprego (MTE) ${ }^{\mathrm{f}}$. Algumas NRs, como a NR 33, que regula o trabalho em espaço confinado, e a NR 35, que regulamenta o trabalho em altura, abordam os fatores e riscos psicossociais relacionados ao trabalho $28-31$.

Por exemplo, o Guia Técnico da NR $33^{28}$ afirma que os riscos psicossociais exercem "influência na saúde mental dos trabalhadores, provocada pelas tensões da vida diária, pressão do trabalho e outros fatores adversos” (p. 54). Já o Manual de auxílio na interpretação e aplicação da norma reguladora $n^{\circ} 35$ : trabalho em altura ${ }^{29}$ considera que:

Os fatores psicossociais relacionados ao trabalho podem ser definidos como aquelas características do trabalho que funcionam como "estressores", ou seja, implicam em grandes exigências no trabalho, combinadas com recursos insuficientes para o enfrentamento das mesmas. (p.17)

Tanto a NR $33^{30}$ como a NR $35^{31}$ versam sobre a necessidade de considerar os riscos psicossociais na avaliação de saúde dos trabalhadores. A NR $33^{30}$ prevê:

Todo trabalhador designado para trabalhos em espa-
ços confinados deve ser submetido a exames médi-
cos específicos para a função que irá desempenhar,
conforme estabelecem as NRs 07 e 31 , incluindo os
fatores de riscos psicossociais com a emissão do res-
pectivo Atestado de Saúde Ocupacional - ASO. (p. 4)

Para a NR $35^{31}$, é dever do empregador avaliar o estado de saúde do trabalhador, considerando também os fatores psicossociais. Nesse sentido, ao definir os fatores psicossociais relacionados ao trabalho, esta NR, em sua versão comentada, considera que uma avaliação psicológica pode ser recomendável, apesar de não a definir como obrigatória ${ }^{29}$.

f O Ministério do Trabalho, Emprego e Previdência Social foi extinto em janeiro de 2019. Suas pastas foram incorporadas a outros ministérios. 
Nessa mesma direção, alguns órgãos internacionais, governos, sindicatos e empresas têm adotado o diagnóstico dos FRPT como parte dos programas de gestão relacionados à saúde do trabalhador ${ }^{16,20,32}$.

\section{Diagnóstico dos fatores de riscos psicossociais no trabalho e avaliações psicossociais}

Apesar de diversas instituições sinalizarem para a necessidade de considerar o contexto social e de trabalho na avaliação dos FRPT, a maioria dos estudos propõe o uso de ferramentas quantitativas para realizar diagnósticos. Trata-se de questionários validados quanto a suas propriedades psicométricas, pontuadas a partir da escala Likert, que objetivam identificar e mensurar os fatores presentes no ambiente de trabalho na percepção dos sujeitos, ou seja, "encaixar" situações subjetivas em respostas quantificáveis.

Em Portugal, Veloso Neto ${ }^{22}$ propõe uma estratégia integrada de avaliação e gestão de riscos psicossociais no trabalho, em etapas sucessivas: elaboração de plano de ação, revisão pela gestão de topo, comunicação do plano de ação, sensibilização e formação de chefias e representantes dos trabalhadores, identificação de fatores de risco, estimação dos riscos, valoração dos riscos, controle e/ou eliminação de fatores de risco e monitorização das ações implementadas.

Já o ISTAS ${ }^{20}$ considera fundamental a participação dos trabalhadores no processo de identificação dos riscos psicossociais, ressaltando que eles possuem uma experiência que não pode ser substituída pelo conhecimento técnico. O instituto sindical espanhol recomenda a aplicação do Copenhagen Psychosocial Questionnaire (COPSOQ II), última versão desenvolvida por Pejtersen et al. ${ }^{33}$, como forma de diagnosticar os riscos psicossociais no trabalho. O COPSOQ-ISTAS vem sendo utilizado também como referência pelos governos do Chile e Peru.

As avaliações psicossociais individuais e os questionários de diagnóstico coletivo sobre os riscos psicossociais no trabalho como o COPSOQ II e o Health Safety Executive (HSE), do Reino Unido ${ }^{34}$, buscam detectar fatores que podem desencadear estresse na percepção dos trabalhadores, incluindo conflitos interpessoais no trabalho.

No Brasil, Facas ${ }^{35}$ preconiza a adoção de um Protocolo de Avaliação dos Riscos Psicossociais (Proart), que inclui dimensões que envolvem a relação entre trabalhador e organização do trabalho, considerando a organização prescrita do trabalho, estilos de gestão, sofrimento patogênico e riscos psicossociais. O PROART tem como referencial teórico a psicodinâmica do trabalho ${ }^{36}$.

Guimarães, Martins e Botelho ${ }^{37}$ apontam que algumas empresas brasileiras vêm sofrendo fiscalizações do trabalho que exigem a observação dos fatores psicossociais e das recomendações de documentos produzidos e assinados por psicólogos. Segundo as autoras, "o Ministério do Trabalho e Emprego, por meio da NR 33, exige que as empresas que possuem atividades em ambientes confinados submetam seus trabalhadores à avaliação psicossocial”37 (p. 58).

As autoras ${ }^{37}$ elencam uma variedade de estratégias que vêm sendo adotadas no Brasil na realização das avaliações psicossociais e concluem que

Em linhas gerais, as experiências relatadas buscaram
elaborar estratégias e roteiros/modelos para essa ava-
liação, realizadas por psicólogos, nos quais se evi-
denciam a utilização de escalas e questionários que
investigam a presença de sintomas indicativos de
transtornos mentais (Ansiedade, Depressão, TEPT,
TOC, TAB, Fobias) e de instrumentos de rastreamen-
to de transtornos mentais em geral. As abordagens
propõem também investigar aspectos relacionados
à qualidade de vida em geral, aos relacionamentos
interpessoais, relacionamentos sociais dentro e fora
do ambiente de trabalho, eventos de vida, e também
a trajetória profissional e a motivação pela opção de
trabalhar em espaço confinado. (p. 64-65)

Ainda segundo as autoras, as avaliações psicossociais devem ser atreladas a uma estratégia de sensibilização mais ampla e contextualizada nas empresas, de forma a facilitar uma "cultura" de prevenção aos riscos inerentes ao trabalho. Elas concluem que as avaliações contribuem na adoção de medidas preventivas em relação à saúde mental dos trabalhadores, bem como têm papel importante na redução de riscos de acidentes e de índices de presenteísmo, absenteísmo e afastamentos ${ }^{37}$.

Na experiência de realização de avaliações psicossociais no trabalho, a primeira autora deste ensaio sistematizou uma série de práticas que vinham sendo desenvolvidas por psicólogos em atendimento às NRs. Constatou-se na ocasião a existência de uma diversidade de formas e modelos de testes e questionários aplicados nas avaliações psicossociais.

A maioria das avaliações era composta por testes projetivos de personalidade, de avaliação de atenção e escalas de mensuração da dimensão de sociabilidade do indivíduo. Alguns profissionais elaboravam questionários como ferramentas de complementação aos testes e encerravam a avaliação fornecendo um laudo psicológico individual em que constava a orientação para a aptidão ou inaptidão para o trabalho em altura e espaço confinado. 


\section{Limitações dos fatores de riscos psicossociais no âmbito da saúde mental relacionada ao trabalho}

Como se evidenciou nos tópicos anteriores, há um denso constructo teórico conceitual a respeito dos FRPT e sobre as formas de diagnosticá-los, geri-los e avaliá-los. No entanto, com base nas referências adotadas, faz-se necessária uma análise crítica que evidencie limitações e indique caminhos possíveis para uma abordagem ampliada da saúde mental dos trabalhadores.

Laurell e Noriega ${ }^{6}$ afirmam que a principal limitação do conceito de risco na busca por compreender os elementos prejudiciais à saúde do trabalhador reside no fato de que através dele os agentes nocivos são examinados de forma isolada. Assim, considera-se que o termo FRPT contribui para a redução do processo psicossocial em um fator de risco mensurável, tais como os riscos químicos ou físicos.

Desta forma, uma crítica necessária ao termo FRPT e suas formas de avaliação diz respeito à tentativa de enquadrar como fator as características das condições e organização do trabalho, desprezando a análise de aspectos macrossociais e históricos presentes na determinação da realidade de trabalho. $\mathrm{Na}$ forma como o conceito de FRPT vem sendo utilizado, observa-se uma prática de identificação de riscos que se propõe a fixar indicadores genéricos para dimensões do trabalho que se dão de forma processual, não estável ou previsível.

Os trabalhadores são sujeitos históricos e sociais, e seu estado de saúde mental tende a refletir o tempo em que estão inseridos e o modo de produção capitalista, que determina sua forma de agir e trabalhar. Portanto, não se pode isolar estes sujeitos e aspectos específicos de seu trabalho de forma a-histórica e descontextualizada socialmente, tomando os determinantes de saúde/doença como medidas exatas e aplicáveis a todos os sujeitos de forma generalizada.

Essas críticas dialogam com Seligmann-Silva ${ }^{5}$, para quem o processo de adoecimento mental dos trabalhadores merece uma análise ampliada, a partir de diversos elementos ou patamares que compõem a realidade de trabalho, considerando aspectos que vão da macroestrutura social a características individuais.

A ideia de identificação de anormalidades e gestão do risco se aproxima de pressupostos da saúde ocupacional que, segundo $\mathrm{Lacaz}^{7}$, atuam com uma visão descolada da historicidade e da dialética dos processos que emergem do modo de produção capitalista, no conflito capital-trabalho:

\begin{abstract}
A abordagem das relações trabalho e saúde-doença parte da ideia cartesiana do corpo como máquina, o qual expõe-se a agentes/fatores de risco [...]. Aqui, os "limites de tolerância" e "limites biológicos de exposição", emprestados da higiene industrial e toxicologia, balizam a intervenção na realidade laboral, buscando "adaptar" ambiente e condições de trabalho a parâmetros preconizados para a média dos trabalhadores normais quanto à suscetibilidade individual aos agentes/fatores ${ }^{7}$. (p. 757)
\end{abstract}

Os testes psicológicos aplicados nas avaliações psicossociais em geral não contemplam o trabalho e seus determinantes e não consideram as condições de trabalho e o processo de produção e gestão. Ou seja, os testes parecem estar distantes das situações de trabalho vivenciadas pelos trabalhadores e, portanto, não captam a complexidade dos processos psicossociais vivenciados no ambiente laboral. Mesmo quando aplicados de maneira coletiva, a avaliação final permanece restrita aos perfis individuais.

Considera-se ainda que as avaliações psicossociais reforçam a cultura higienista de separação entre "normais" e "anormais" ou "aptos" e "inaptos" para atuar em determinadas atividades laborais, conferindo a adaptação dos sujeitos, que teriam perfis mais ou menos "resilientes". De acordo com Seligmann-Silva ${ }^{5}$, a saúde mental não pode ser confundida com o processo de "adaptação", sendo necessário considerar a perda processual e silenciosa provocada pelas formas de organização e condições de trabalho, processo que a autora denomina de "desgaste mental" dos trabalhadores.

Ainda que remetam a aspectos das condições e organização do trabalho, as definições encontradas na literatura sobre os FRPT - ao considerar a "percepção subjetiva" do sujeito sobre estes determinantes ${ }^{26}$ ou a interação entre aspectos e condições de trabalho com a capacidade e necessidades dos trabalhadores, bem como a forma como esses elementos são percebidos pelo sujeito ${ }^{16}$ - reforçam uma concepção limitada, que pode resultar na culpabilização do trabalhador pelo seu processo de adoecimento. Estas definições se viabilizam dentro de um contexto ideológico determinado, que inclui a responsabilização do trabalhador, na medida em que a forma como ele "percebe" ou tem "capacidade de trabalhar ou atender a demandas" é que vai influenciar em seu adoecimento, e não a precariedade das condições, organização e formas de gestão do trabalho, imersas dialética e assimetricamente no modo de produção capitalista.

Problematiza-se o tipo de enfoque daqueles que se aproveitam das confusões conceituais e das limitações do termo FRPT, como muitas empresas que têm contratado profissionais para realizar avaliações psicossociais, mas sem se comprometer com a transformação das condições e organização do trabalho. Enfatiza-se 
o reducionismo de considerar um processo dinâmico e contínuo por meio de fatores específicos, medidos objetivamente. Neste sentido, muitas vezes os FRPT são apontados genericamente como fatores estressores na percepção dos indivíduos, de modo a responsabilizá-los para que adotem estratégias individuais de defesa (conhecidas como coping ${ }^{38}$ ) ou outros mecanismos que aumentem a resiliência individual.

Clot $^{39}$ alerta que algumas mudanças advindas da gestão dos riscos psicossociais no trabalho podem inclusive piorar o quadro de saúde dos trabalhadores, ao adotar uma postura higienista, que coloca em evidência a fragilidade das pessoas. Corre-se o risco de restringir o diagnóstico dos FRPT nas organizações a intervenções coletivas sem a participação efetiva dos trabalhadores, ou implantar ações no âmbito individual - como a mudança dos trabalhadores de local de trabalho devido a conflitos interpessoais - sem considerar que na origem destes conflitos desenvolvem-se mecanismos de pressão, poder e insegurança, tanto na atividade do trabalhador quanto do gestor. Desconsidera-se o que está na origem desses conflitos, bem como as determinações macrossociais que interferem nas práticas cotidianas de trabalho nas empresas.

Para Bernardo et al. ${ }^{4}$, a inclusão dos riscos psicossociais nas preocupações governamentais de diferentes países e agências internacionais representa um grande avanço. No entanto, os autores enfatizam que a maneira como vêm se configurando estas ações - a partir de um diagnóstico que foca relações interpessoais, fatores individuais e alguns elementos da organização do trabalho - não modifica aspectos técnicos que têm consequências diretas para a saúde dos trabalhadores.

\section{Considerações finais}

Parece haver uma confusão de conceitos, incluindo as avaliações psicossociais, que se aproximam mais da avaliação psicológica e menos das dimensões psicossociais que envolvem o trabalho. Então, o que de fato seria realizar uma avaliação psicossocial? Pode esta avaliação ter como alvo um sujeito singular, desconsiderando as dimensões concretas da realidade que o constituem, bem como suas determinações históricas? Há que se considerar, ainda, o processo produtivo e de valoração do trabalho nas determinações de saúde/ doença dos trabalhadores, superando o modelo hegemônico de indicadores de risco como algo isolado, descolado da dinâmica global do processo de trabalho ${ }^{6}$.

Seligmann-Silva ${ }^{5}$ defende que para compreender o processo de adoecimento mental dos trabalhadores é necessário considerar cinco diferentes patamares:
1) A divisão internacional da riqueza, com suas questões macrossociais, macroeconômicas e de políticas internacionais que interferem direta ou indiretamente nas exigências encontradas pelos trabalhadores.

2) O contexto nacional, com suas políticas públicas e leis que garantem direitos aos trabalhadores, proteção do emprego e ações de promoção da saúde dos cidadãos.

3) O contexto das empresas, com seus valores éticos, suas situações de trabalho, formas de gestão e maneiras de manter o controle sobre os trabalhadores.

4) A qualidade dos espaços microssociais de trabalho, que são responsáveis pelas interações sociais e pelo sentimento de coletividade dos trabalhadores.

5) A compreensão das características individuais, da subjetividade e da trajetória de vida dos indivíduos.

Ou seja, é necessário considerar os diversos elementos políticos, econômicos, sociais, culturais, ambientais e intrapsíquicos no processo de saúde/ doença dos trabalhadores.

Maeno e Paparelli ${ }^{40}$ também defendem a necessidade de compreender os fatores multicausais presentes no processo de desgaste mental dos trabalhadores, considerando as condições e formas de organização do trabalho. Para as autoras, é necessário levar em conta, entre outros elementos, aspectos que envolvem a relação de poder nas organizações, o controle do tempo e das atividades, exigências e metas.

Ainda nesta direção, Souza ${ }^{41}$ afirma que os diversos atores sociais, por meio de seu preparo técnico, sensibilidade e compromisso social, precisam estar atentos às demandas apresentadas pela população trabalhadora e procurar promover, de forma criteriosa, a saúde psíquica, minimizando os grandes impactos provocados pela complexidade das organizações e situações de trabalho existentes na atualidade. Esse caminho é bem mais complicado que o trilhado atualmente, mas seus resultados são certamente muito mais eficientes e engajados com a realidade social.

Defende-se que os trabalhadores devam participar no planejamento das ações de combate ao adoecimento mental, pois são eles quem vivenciam a realidade de trabalho e suas consequências para a saúde. O ISTAS ${ }^{20}$ parte dessa perspectiva, ao reivindicar a participação dos trabalhadores nesse processo. No entanto, compreendemos que a aplicação do questionário proposto não evidencia as relações de dominação no trabalho, inclusive na medida em 
que o mesmo instrumento pode ser aplicado nos mais diferentes contextos laborais.

Diante desse contexto, defende-se a necessidade de um olhar crítico para o conceito "fatores de riscos psicossociais", que vem sendo utilizado de forma genérica como fator estressor, em uma perspectiva ideológica determinada, bem como para as práticas de avaliação psicossocial. Na medida em que são identificadas suas limitações, tenta-se dar visibilidade ao complexo cenário do mundo do trabalho e às diferentes determinações e condicionantes do processo saúde e doença no trabalho.
Desta forma, embora a criação e utilização de instrumentos de avaliação dos FRPT coloquem em evidência pautas que eram totalmente negligenciadas, como as condições e organização do trabalho, é preciso problematizar os limites do termo e dos instrumentos utilizados para avaliar as dimensões psicossociais presentes no ambiente de trabalho. Há de se considerar os atuais contextos sociais e ideológicos, realizando uma leitura ampla e crítica que aponte tais limitações, visto que a tentativa de simplificar e de identificar os FRPT, inclusive deixando sua gestão nas mãos das próprias organizações, reflete um cenário de ocultamento ou suavização do conflito capital-trabalho.

\section{Contribuições de autoria}

Pereira ACL e Iguti AM participaram da concepção do projeto, levantamento e análise das informações e, juntamente com Souza HA e Lucca SR, contribuíram com a redação, revisão do manuscrito e aprovação do texto final para publicação. Todos os autores assumem integral responsabilidade pelo trabalho e o conteúdo publicado.

\section{Referências}

1. Watanabe EMB. Aspectos psicossociais de risco no trabalho e a saúde mental dos carteiros da cidade de São Paulo [dissertação]. São Paulo: Fundacentro; 2015.

2. Ribeiro MFA. Riscos psicossociais no trabalho dos enfermeiros [dissertação]. Viseu: Escola Superior de Saúde de Viseu; 2015.

3. Souza SF, Carvalho FM, Araújo TM, Porto LA. Fatores psicossociais do trabalho e transtornos mentais comuns em eletricitários. Rev Saude Pública. 2010;44(4):710-7.

4. Bernardo MH, Souza HA, Pinzón JG, Kawamura EA. Salud Mental relacionada com el trabajo: desafios para las políticas públicas. Univ Psychol. 2016;14(5):1613-23.

5. Seligmann-Silva E. Trabalho e desgaste mental: o direito de ser dono de si mesmo. São Paulo: Cortez, 2011.

6. Laurell AC, Noriega M. Processo de produção e saúde: trabalho e desgaste operário. São Paulo: Hucitec, 1989.

7. Lacaz FAC. O campo saúde do trabalhador: resgatando conhecimentos e práticas sobre as relações trabalho-saúde. Cad Saude Pública. 2007;23(4):757-66.

8. Canguilhem G. O normal e o patológico. Rio de Janeiro: Forense Universitária, 1990.

9. Antunes R, Praun L. A sociedade dos adoecimentos no trabalho. Serviço Soc Soc. 2015;123:407-27.

10. Bernardo MH. Trabalho duro, discurso flexível: uma análise das contradições do toyotismo a partir da vivência de trabalhadores. São Paulo: Expressão Popular, 2009.
11. Franco T, Druck G, Seligmann-Silva E. As novas relações de trabalho, o desgaste mental do trabalhador e os transtornos mentais no trabalho precarizado. Rev Bras Saude Ocup. 2010;35(122):229-48.

12. Gaulejac V. Gestão como doença social: ideologia, poder gerencialista e fragmentação social. Aparecida: Ideias e Letras; 2007.

13. Abílio LC. Uberização do trabalho: subsunção real da viração. Passa Palavra [Internet]. 2017 fev 19 [citado em 20 out 2017]; Ideias e Debates. Disponível em: http://passapalavra. info/2017/02/110685

14. Brasil. Adoecimento mental e trabalho: a concessão de benefícios por incapacidade relacionados a transtornos mentais e comportamentais entre $2012 \mathrm{e}$ 2016 [Internet]. Brasília, DF: Ministério da Fazenda, 2017 [citado em 4 jul 2020]. Disponível em: http:// sa.previdencia.gov.br/site/2017/04/1\%C2\%BAboletim-quadrimestral.pdf

15. Lopez AD, Murray CC. The global burden of disease, 1990-2020. Nat Med. 1998; 4(11):1241-3.

16. International Labour Organisation. Psychosocial factors at work: recognition and control [Internet]. Geneva: International Labour Office; 1986 [citado em 2 mar 2017]. Disponível em: http://www.ilo.org/ public/libdoc/ilo/1986/86B09_301_engl.pdf

17. World Health Organization. PRIMA-EF: guidance on the European framework for psychosocial risk management: a resource for employers and worker representatives [Internet]. Geneva: WHO; 2008 [citado em 12 maio 2017]. Disponível em: http://apps.who.int/iris/ bitstream/10665/43966/1/9789241597104_eng_ Part1.pdf 
18. International Labour Organization. Workplace stress: a collective challenge Internet]. Geneva: International Labour Organization; 2016 [citado em 5 jul 2020]. Disponível em: https://www.ilo.org/wcmsp5/groups/ public/---ed_protect/---protrav/---safework/documents/ publication/wcms_466547.pdf

19. International Labour Organization. Psychosocial risks and work-related stress [Internet]. Geneva: International Labour Orgazation (ILO): c1996-2020 [citado em 5 jul 2020]. Disponível em: https://www. ilo.org/global/topics/safety-and-health-at-work/ areasofwork/workplace-health-promotion-and-wellbeing/WCMS_108557/lang--en/index.htm

20. Instituto Sindical de Trabajo, Ambiente y Salud. Organización del Trabajo, Salud y Riesgos Psicosociales: guía para la intervención sindical [Internet]. Madrid: ISTAS, 2006 [citado em 12 mar 2017]. Disponível em: http://www.istas.net/web/ abreenlace.asp?idenlace $=3362$

21. Gollac M, Bordier M. Mesurer les facteurs psychosociaux de risque au travail pour les maîtriser: rapport du Collège d'expertise sur le suivi des risques psychosociaux au travail, faisant suite à la demande du Ministre du travail, de l'emploi et de la santé. Paris: Collège d'Expertise sur le Suivi des Risques Psychosociaux au Travail; 2011. [citado em 2017 jun 10]. Disponível em: https://travailemploi.gouv.fr/IMG/pdf/rapport_SRPST_definitif_ rectifie_11_05_10.pdf

22. Veloso Neto H. Estratégias organizacionais de gestão e intervenção sobre riscos psicossociais no trabalho. International Journal on Working Conditions [Internet]. 2015 [citado em 18 ago 2017];9:1-21. Disponível em: http://ricot.com.pt/artigos/1/IJWC.9_ HVN.p.1-21.pdf

23. Alcántara SM, Sánchez AH. Necesidad de estudios y legislación sobre factores psicosociales en el trabajo. RCSP. 2005;31(4):338-44.

24. Álvarez Briceño P. Los riesgos psicosociales y su reconocimiento como enfermedad ocupacional: consecuencias legales y económicas. Telos. 2009;11(3):367-85.

25. Baruki LV. Riscos psicossociais e saúde mental do trabalhador: por um regime jurídico preventivo. São Paulo: LTR, 2015.

26. Brasil. Instrução Normativa DC/INSS no 98, de 5 de dezembro de 2003. Aprova Norma Técnica sobre Lesões por Esforços Repetitivos - LER ou Distúrbios Osteomusculares Relacionados ao Trabalho - DORT. Diário Oficial da União. 10 dez 2003.

27. Guimarães LAM. Fatores psicossociais de risco no trabalho. $2^{\circ}$ Congresso Internacional sobre Saúde Mental no Trabalho; 12-14 out 2006; Goiânia, Brasil. Goiânia: Fórum de Saúde e Segurança do Trabalho do Estado de Goiás; 2016.

28. Brasil. Ministério do Trabalho e Emprego. Guia técnico da NR-33. Brasília, DF: Ministério do Trabalho e Emprego; 2013.

29. Brasil. Ministério do Trabalho e Emprego. Manual de auxílio na interpretação e aplicação da Norma
Reguladora $\mathrm{n}^{\circ}$ 35: trabalho em altura. Brasília, DF: Ministério do Trabalho; 2018 [citado em 5 jul 2020]. Disponível em: https://enit.trabalho.gov.br/ portal/images/Arquivos_SST/SST_Publicacao_e Manual/CGNOR---MANUAL-CONSOLIDADE-DANR-35.pdf

30. Brasil. Ministério do Trabalho e Emprego. NR33 segurança e saúde nos trabalhos em espaços confinados [Internet]. Brasília, DF: Ministério do Trabalho e Emprego; 2012 [citado em 5 jul 2020]. Disponível em: https:/enit.trabalho.gov.br/portal/ images/Arquivos_SST/SST_NR/NR-33.pdf

31. Brasil. Ministério do Trabalho e Emprego. NR 35: trabalho em altura [Internet]. Brasília, DF: Ministério do Trabalho e Emprego; 2012 [citado em 5 jul 2020]. Disponível em: https:// enit.trabalho.gov.br/portal/images/Arquivos_SST/ SST_NR/NR-35.pdf

32. Ferreira ABR. Saúde no trabalho: uma avaliação de riscos psicossociais numa empresa do ramo industrial [dissertação]. Porto: Universidade Fernando Pessoa, 2015.

33. Pejtersen JH, Kristensen TS, Borg V, Bjorner JB. The second version of the Copenhagen Psychosocial Questionnaire. Scand J Public Health. 2010;38(3):8-24.

34. Edwards JA, Webster S, Van Laar D, Easton S. Psychometric analysis of the UK Health and Safety Executive's Management Standards work-related stress Indicator Tool. Work Stress. 2008;22(2):96-107.

35. Facas EP. Protocolo de avaliação dos riscos psicossociais no trabalho: contribuições da psicodinâmica do trabalho [tese]. Brasília, DF: Universidade de Brasília, 2013.

36. Dejours C, Abboucheli E, Jayet C. Psicodinâmica do trabalho: contribuição da escola dejouriana à análise da relação prazer, sofrimento e trabalho. São Paulo: Atlas, 1994.

37. Guimarães LAM, Martins DA, Botelho ASO. Contribuições para a avaliação psicossocial da Norma Reguladora 33 (NR-33). Perspectivas em Gestão \& Conhecimento 2013;Número Especial 3:57-66.

38. Lazarus R, Folkman, S. Coping and adaptation. In: Gentry WD, editor. Handbook of Behavioral Medicine. New York: The Guilford Press; 1984. p. 282-325.

39. Clot Y. Le travail à coeur: pour en finir avecles risques psychosociaux. Paris: La Découverte/Poche, 2015.

40. Maeno M, Papparelli R. O trabalho como ele é e a saúde mental do trabalhador. In: Silveira MA. Inovação para desenvolvimento de organizações sustentáveis: trabalho, fatores psicossociais e ambiente saudável. Campinas: Centro de Tecnologia da Informação Renato Archer; 2013. p. 145-166.

41. Souza HA. Saúde mental relacionada ao trabalho na rede pública de saúde brasileira: concepções e atuações transformadoras [doutorado]. Campinas: Pontifícia Universidade Católica; 2017. 